\title{
Patient Outcomes, Cost, and Length of Stay Associated With Biventricular Assist Devices in the United States (2009-2015)
}

\author{
Hyeon Ju Ali ${ }^{1}$, Afshin Ehsan ${ }^{2}$, Kevin Kennedy ${ }^{3}$, Peter Riley ${ }^{4}$, Angie $\mathrm{Seo}^{2}$, Navkaranbir \\ Bajaj $^{5}$, Amitoj Singh ${ }^{6}$, Daniel Levine ${ }^{2}$, Michael Kiernan ${ }^{7}$, Frank Sellke ${ }^{2}$, and Rayan \\ Yousefzai $^{1}$ \\ ${ }^{1}$ Houston Methodist Hospital \\ ${ }^{2}$ Brown University Warren Alpert Medical School \\ ${ }^{3}$ Saint Luke's Mid America Heart Institute \\ ${ }^{4}$ University of Maryland Medical System \\ ${ }^{5}$ The University of Alabama System \\ ${ }^{6}$ The University of Arizona College of Medicine Tucson \\ ${ }^{7}$ Tufts Med Ctr
}

May 29, 2021

\begin{abstract}
Objectives: With recent advances in left ventricular assist device (LVAD) technology, the utilization of LVADs has increased while mortality, cost, and length of stay (LOS) have significantly decreased. This study sought to determine the national trends of surgically implanted biventricular assist devices (BiVADs). Methods: This is a retrospective analysis of the National Inpatient Sample (NIS) database for internal BiVADs $(\mathrm{n}=538)$ and external BiVADs $(\mathrm{n}=727)$ from 2009 to 2015 . Results: Utilization of all surgically implanted BiVADs decreased (4.8\% per year, $\mathrm{p}<0.001)$, and was driven by a decline in utilization of external BiVADs $(9.4 \%$ per year, $\mathrm{p}<0.001)$. There was no significant change in BiVAD-associated mortality. However, cost and LOS increased significantly, and these trends were isolated to patients who died during their hospitalization. Patients with internal BiVADs incurred higher cost than external BiVAD patients but were more likely to survive. Conclusions: The decline in surgically implanted BiVAD utilization may be due to an improvement in LVAD technology as well as an increase in the availability of percutaneous temporary circulatory support. Future prospective studies are needed to compare various BiVAD configuration strategies and to optimize BiVAD patient selection and perioperative care.
\end{abstract}

\section{Introduction}

From 2009 to 2015, in-hospital mortality and complication rates associated with left ventricular assist device (LVAD) implantation significantly declined. ${ }^{1,2}$ Much of the favorable outcomes have been attributed to the evolution of mechanical circulatory support from pneumatic mechanical-bearing pulsatile-flow (PF) pumps to magnetically-levitated continuous-flow (CF) devices. According to the latest results from the MOMENTUM 3 trials, $77 \%$ of patients receiving the HeartMate III LVAD had a stroke-free 2-year survival. ${ }^{3}$ Despite these encouraging outcomes, right ventricular failure (RVF) post-LVAD remains a significant cause of adverse outcomes - in some instances requiring biventricular assist devices (BiVADs). ${ }^{4}$

Despite the advances in LVAD technology, most BiVADs - including total artificial hearts (TAHs) and external BiVADs - are still pneumatic PF devices. BiVAD configurations are diverse and include completely internalized devices - such as the TAH or two separate internal ventricular assist devices (VADs) - and external support, such as paracorporeal PF BiVADs, and CF centrifugal pumps (e.g., CentriMag). ${ }^{5}$ Given 
these fundamental differences, it is unknown whether the promising national trends in the LVAD population - improvement in survival, decrease in length of stay (LOS), and reduction in complication rates - also hold true for patients receiving surgically implanted BiVADs. This study is the first to describe the national trends in utilization, mortality, complications, cost, and LOS for patients receiving BiVADs.

\section{Materials and methods}

\subsection{Study design}

This is a retrospective analysis of the National Inpatient Sample (NIS) database. The NIS is reflective of all hospital admissions in the United States. It serves as an essential tool for analyzing the trends in outcomes and costs of healthcare procedures. More details regarding the database are available online. ${ }^{5}$ Given the data from NIS are de-identified, this study was exempt from Institutional Review Board review. Consent statement and clinical trial registration are not applicable for this study.

\subsection{Study population and outcomes}

The NIS database was queried to identify the hospitalizations of the following population: 1) adult patients age [?] 18 years old and 2) those with implantation of surgically implanted internal BiVADs (International Classification of Disease $9^{\text {th }}$ revision (ICD-9) code 37.52 , which includes TAH) or surgically implanted external BiVADs (ICD-9 code 37.60). The patients with orthotropic heart transplantations (ICD-9 code $37.5,37.51$, and 33.6), temporary non-implantable external systems (ICD-9 code 37.62), single ventricular devices (ICD-9 code 37.65 and 37.66), as well as percutaneous external devices (ICD-9 code 37.68) were excluded. Of note, the use of two separate surgically implanted VADs used for biventricular support fell ICD coding for "single ventricular devices" and thus could not be isolated and captured in our analysis. We also excluded the patients receiving intra-aortic balloon pumps (IABPs, ICD-9 code 37.61 and 37.68) as well as implantable heart systems (ICD-9 code 37.66). In total, 1,264 patients met these criteria, representing 21,204 patients nationwide.

Primary outcomes of interest were utilization and in-hospital mortality. Secondary outcomes included LOS, cost of hospitalization, and complications: major bleeding requiring transfusion, gastrointestinal (GI) bleed, hemoperitoneum, intracranial bleed, hemolytic anemia, vascular complications, acute renal failure, mechanical complications, systemic embolism, stroke or transient ischemic attack (TIA), and infections. In addition to demographic information, other relevant comorbidities (e.g., coronary artery disease (CAD), myocardial infarction (MI), chronic pulmonary disease, anemia, coagulopathy, diabetes, hypertension, renal failure, and peripheral vascular disease) were queried. Information regarding the hospitalization - the region of hospital, location and teaching status, volume, elective vs. non-elective implantation, and insurance status - were included in the analyses.

\subsection{Statistical analysis}

The baseline characteristics of patients with internal and external BiVAD were compared using the $\chi^{2}$ test for categorical variables and analysis of variance for continuous variables. The Poisson regression model was used to assess the change in estimated quarterly volumes of internal, external, and all BiVADs. Finally, we sought to explain the national trends using exploratory subgroup analyses. The total population was divided into patients who survived the index hospitalization and those who died during the hospitalization. We performed regression analyses using comorbidities and complications as covariates. STATA was used to perform all analyses. Statistical significance was considered to be $p<0.05$.

\section{Results}

A total of 21,204 patients received either surgically implanted internal or external BiVADs between 2009 and 2015. The mean age of the population was 51.2 years (standard deviation or SD $=14.4$ ) at the time of implantation (see Table 1). Approximately one quarter $(25.1 \%)$ of all patients were female, and two thirds $(63.7 \%)$ were Caucasians. Almost half of the patients $(48.4 \%)$ had private insurance, and $30.9 \%$ had 
Medicare coverage. Most implantations took place in large (86\%), urban teaching hospitals (90.3\%), and $18.5 \%$ of them occurred as elective admissions.

\subsection{Time trends in utilization, mortality, cost, and LOS among BiVAD patients}

Between 2009 and 2015, there was an overall decline in the utilization of all BiVADs $(-4.8 \%$ per year, $p<$ 0.001 , see Figure 1). There was a significant increase in the use of internal BiVADs $(+10.0 \%$ per year, $p$ $<0.001$, see Figure 1) and a significant decrease in the use of external BiVADs $(-9.4 \%$ per year, $p<0.001$, see Figure 1). There were no significant changes in mortality for all BiVADs, internal BiVADs, or external BiVADs (see Appendix A). The costs associated with BiVAD implantation increased significantly $(+23.0 \%$ per year, $p<0.001)$. The LOS also had an increasing trend, although not significant $(p=0.083$, see Figure 2 ). Among complications associated with BiVAD implantations, the rates of hemoperitoneum $(p=0.003)$, gastrointestinal (GI) bleed $(p=0.001)$, and acute renal failure $(p=0.008)$ increased significantly over time (see Table 2). Among known comorbidities, CAD $(p=0.006)$, MI $(p=0.010)$, fluid and electrolyte disorders $(p=0.043)$, and history of renal failure $(p=0.011)$ increased over time (see Table 2$)$.

\subsection{Time trend analysis for cost and LOS among BiVAD patients: the survivors versus deceased}

To explain the increase in cost and LOS among BiVAD patients despite no changes in mortality, a time trend analysis was performed. Stratification based on internal vs. external BiVADs demonstrated no significant time trends in cost and LOS for either internal or external BiVADs. Stratification based survival of the index hospitalization demonstrated no significant time trend in LOS or cost among those that survived, however, there was a significant and sizeable increase in $\operatorname{LOS}(+52.0 \%$ per year, $\mathrm{p}=0.002$, see Figure 2$)$, as well as cost $(+15 \%$ per year, $\mathrm{p}<0.001$, see Figure 3$)$ among those that died. After controlling for known complications and comorbidities outlined in Table 2 , LOS $(+3.8 \%$ per year, $\mathrm{p}=0.025)$ and cost $(+\$ 33,736$ per year, $\mathrm{p}=$ 0.002 ) increased significantly over time among the patients who died during their hospitalization.

3.3 Comparisons between internal and external BiVADs: utilization, mortality, LOS, cost, and complications

Internal BiVADs were implanted in $538(42.5 \%)$ patients and external BiVADs in $727(57.5 \%)$ patients (see Table 3). More patients were female in the external BiVAD group ( $30.1 \%$ vs $18.4 \%, p=0.024)$. There were no significant differences between internal and external BiVADs in insurance coverage, size, and teaching status of the hospitals, as well as elective cases. More of the external BiVAD implantation occurred in the Northeast compared to the internal BiVADs $(36.0 \%$ vs. $14.9 \%, p=0.002)$. Internal BiVAD implantations were more likely than external BiVAD implantations to take place in urban teaching hospitals (98.3\% vs. $90.3 \%, p<0.001)$. Significantly more patients in the external BiVAD group had documented history of CAD $(44.9 \%$ vs. $22.1 \%, p<0.001)$, pulmonary circulatory disorders $(5.3 \%$ vs $0, p=0.014)$, and valvular disease $(7.2 \%$ vs. $0, p=0003)$. History of renal failure was more prevalent in the internal BiVAD group (36.1\% vs. $16.1 \%, p<0.001)$.

Higher mortality was observed among patients with external BiVADs compared to those with internal BiVADs $(59.1 \%$ vs. $45.4 \%, p=0.022$, see Table 3$)$. Internal BiVADs were associated with longer median LOS (64.0 vs. 20.0 days, $p<0.001)$ and higher cost (\$468,321 vs. $\$ 208,809$ per hospitalization, $p<0.001)$. There were more complications of acute renal failure $(85.6 \%$ vs. $74.0 \%, p=0.020)$ and hemolytic anemia $(4.5 \%$ vs. $0.7 \%, p=0.038)$ among those with internal BiVADs. There were no significant differences in rates of bleeding, infection, or stroke.

\section{Discussion}

This study is the first to describe national trends in utilization, mortality, LOS, and cost for patients receiving surgically implanted internal and external BiVADs. From 2009 to 2015, the utilization of all BiVADs nationwide decreased, driven by a decrease in utilization of external BiVADs. Compared to the significant decline in mortality and cost associated with LVAD implantation during this period, ${ }^{1,2}$ our analysis demonstrated no change in mortality and a rise in cost and LOS associated with BiVADs. The unchanged 
mortality for BiVAD patients was coupled with an increase in the burden of comorbidities. While LOS and the cost associated with survivors stayed the same, LOS and cost related to the deceased patients increased, even after controlling for all comorbidities and complications. These findings may suggest that we have also evolved in our capability of caring for higher-risk patients utilizing BiVADs although this has required higher costs caring for the sickest patients who eventually died during the hospitalization.

The decline in surgically implanted BiVAD utilization was driven by a decrease in utilization of external BiVADs, which outnumbered the increase in utilization of internal BiVADs. Our finding correlated with one other prior study that used the UNOS database, which showed a decrease in utilization of PF-BiVADs from 2010 to $2013 .{ }^{6}$ There are several potential reasons for the decline in BiVAD utilization. First, the availability of percutaneous biventricular MCS, such as ECMO, Impella RP, and ProtekDuo, may be obviating the need for surgically placed BiVADs. Second, LVAD utilization has significantly increased during this period. ${ }^{1,2}$ Earlier implantation of LVADs leads to decompression of the left ventricle and a decrease in pulmonary pressures and subsequent afterload on the RV. The latter may therefore reduce the incidence of RVF and decrease the need for BiVADs.

This is the first study to directly compare outcomes of surgically implanted internal and external BiVADs. Patients with internal BiVADs had significantly longer LOS and higher cost of hospitalizations compared to that of external BiVADs; however, the mortality was lower in patients with internal BiVADs. Significantly more of the internal BiVAD implantations occurred in urban teaching hospitals. Prior analysis of the INTERMACS database showed that patients receiving BiVADs at higher volume centers ( $>10$ implantations per year) had a better chance of 1-year survival (71\% vs. $53 \%, p=0.003) .{ }^{7}$ As a result, the lower mortality seen in internal BiVAD patients may be the result of higher implant volumes seen at urban teaching hospitals compared to rural or non-teaching facilities. More studies are needed to better understand the differences in mortality, LOS, and cost between various BiVAD configurations - specifically with regards to patient selection, implant center volume, and rates of complications.

Despite the advancement of LVAD technology and patient care, RVF after LVAD placement remains a significant source of complication - up to $3.9-14.5 \%$ according to the recent multi-national studies. ${ }^{89}$ Prior studies have demonstrated that planned, and earlier BiVAD implantation is associated with higher survival rates. ${ }^{10,11}$ Other studies have demonstrated that angiotensin-converting enzyme inhibitors (ACEI) can significantly reduce the risk of GI bleeds associated with LVAD implantation ${ }^{12}$ and improve mortality. ${ }^{13}$ However, it is unclear whether these findings have led to changes in practice. Future investigation should focus on implantation of evidence-based strategies for practical improvements in the care of patients with BiVADs.

This study used ICD-9 codes to identify the population that received BiVADs, and thus, have limitations inherent to the coding system. For example, the off-label use of two separate surgically implanted VADs used for biventricular support was classified as two single ventricular devices (ICD-9 37.65), and thus, were excluded from our study. Consequently, our study captures only a subset of internal BiVADs, and likely underestimates the total number of internal and all BiVADs. Despite this limitation, our study demonstrated a significant rise in internal BiVAD utilization during the study period. Future studies are needed to assess and compare the outcomes of this particular configuration of biventricular support. This study also does not include many other types of MCS, such as paracorporeal devices and other external systems. Outcomes of other temporary systems of circulatory support should be considered in a different forum, and is an important area for future investigation.

Our study was limited to ICD-9 codes, which capture only three-quarters of the hospitalizations in 2015 (switched to ICD-10 thereafter). We addressed this limitation by evaluating time trends based on quarterly numbers. One of the limitations of the NIS database is that it does not allow for stratification of the data based on a center's volume of BiVAD implantations. Moreover, given that most centers that perform BiVADs were large-volume centers, this analysis was not powered to detect differences based on implantation experience. As an exploratory analysis, our study tested several hypotheses, which likely reduced the power of the study. This study is retrospective and our findings should be confirmed with prospective trials when possible. 


\section{Conclusion}

From 2009 to 2015, BiVAD utilization decreased, and the associated costs increased, while LVAD utilization increased, and the related cost and mortality decreased. While the mortality rate among BiVAD patients did not change, the patient population had an increasing burden of comorbidities during this time. Compared to patients with external BiVADs, patients with internal BiVADs had longer LOS and higher costs associated with their hospitalizations, but were also more likely to survive. More studies regarding the optimal BiVAD configurations are needed to determine appropriate patient selection and perioperative care to improve outcomes and cost-efficiency.

\section{Bibliography}

1. Briasoulis A, Inampudi C, Akintoye E, et al. Regional Variation in Mortality, Major Complications, and Cost After Left Ventricular Assist Device Implantation in the United States (2009 to 2014). Am J Cardiol . 2018;121(12):1575-1580. doi:10.1016/j.amjcard.2018.02.047

2. Shah N, Agarwal V, Patel N, et al. National Trends in Utilization, Mortality, Complications, and Cost of Care after Left Ventricular Assist Device Implantation from 2005 to 2011. Ann Thorac Surg . 2016;101(4):1477-1484. doi:10.1016/j.athoracsur.2015.09.013

3. Mehra MR, Uriel N, Naka Y, et al. A Fully Magnetically Levitated Left Ventricular Assist Device Final Report. N Engl J Med . Published online 2019:NEJMoa1900486. doi:10.1056/NEJMoa1900486

4. Dang NC, Topkara VK, Mercando M, et al. Right heart failure after left ventricular assist device implantation in patients with chronic congestive heart failure. J Heart Lung Transplant . 2006;25(1):1-6. doi:10.1016/j.healun.2005.07.008

5. Briasoulis A, Akintoye E, Mohsen A, et al. Trends in utilization, mortality, major complications, and cost after total artificial heart implantation in the United States (2009-2015). Hell J Cardiol . 2019;(xxxx). doi:10.1016/j.hjc.2019.02.002

6. Levin AP, Jaramillo N, Garan AR, et al. Outcomes of contemporary mechanical circulatory support device configurations in patients with severe biventricular failure. J Thorac Cardiovasc Surg . 2016;151(2):530535.e2. doi:10.1016/j.jtcvs.2015.10.019

7. Arabía FA, Cantor RS, Koehl DA, et al. Interagency registry for mechanically assisted circulatory support report on the total artificial heart. J Hear Lung Transplant . 2018;37(11):1304-1312. doi:10.1016/j.healun.2018.04.004

8. Kiernan MS, Wilson Grandin E, Brinkley M, et al. Early Right Ventricular Assist Device Use in Patients Undergoing Continuous-Flow Left Ventricular Assist Device Implantation: Incidence and Risk Factors from the Interagency Registry for Mechanically Assisted Circulatory Support. Circ Hear Fail . 2017;10(10). doi:10.1161/CIRCHEARTFAILURE.117.003863

9. Soliman OII, Akin S, Muslem R, et al. Derivation and validation of a novel right-sided heart failure model after implantation of continuous flow left ventricular assist devices. Circulation . 2018;137(9):891-906. doi:10.1161/CIRCULATIONAHA.117.030543

10. Fitzpatrick JR, Frederick JR, Hiesinger W, et al. Early planned institution of biventricular mechanical circulatory support results in improved outcomes compared with delayed conversion of a left ventricular assist device to a biventricular assist device. J Thorac Cardiovasc Surg . 2009;137(4):971-977. doi:10.1016/j.jtcvs.2008.09.021

11. Shah P, Ha R, Singh R, et al. Multicenter experience with durable biventricular assist devices. J Hear Lung Transplant . 2018;37(9):1093-1101. doi:10.1016/j.healun.2018.05.001

12. Converse MP, Sobhanian M, Taber DJ, Houston BA, Meadows HB, Uber WE. Effect of Angiotensin II Inhibitors on Gastrointestinal Bleeding in Patients With Left Ventricular Assist Devices. J Am Coll Cardiol 
13. Ghashghaei R, Yousefzai R, Liwinski F, Tran H, Adler ED. The Role of Neurohormonal Therapy in Patients with Left Ventricular Assist Devices.J Hear Lung Transplant . 2016;35(4):S396. doi:10.1016/j.healun.2016.01.1143

\section{Hosted file}

BiVAD Table 1.docx available at https://authorea.com/users/416647/articles/524111-patientoutcomes-cost-and-length-of-stay-associated-with-biventricular-assist-devices-in-theunited-states-2009-2015

\section{Hosted file}

BiVAD Table 2_JCardSurg_4.24.21.docx available at https://authorea.com/users/416647/articles/ 524111-patient-outcomes-cost-and-length-of-stay-associated-with-biventricular-assistdevices-in-the-united-states-2009-2015

\section{Hosted file}

BiVAD Table 3_-JCardSurg_4.24.21.docx available at https://authorea.com/users/416647/ articles/524111-patient-outcomes-cost-and-length-of-stay-associated-with-biventricularassist-devices-in-the-united-states-2009-2015

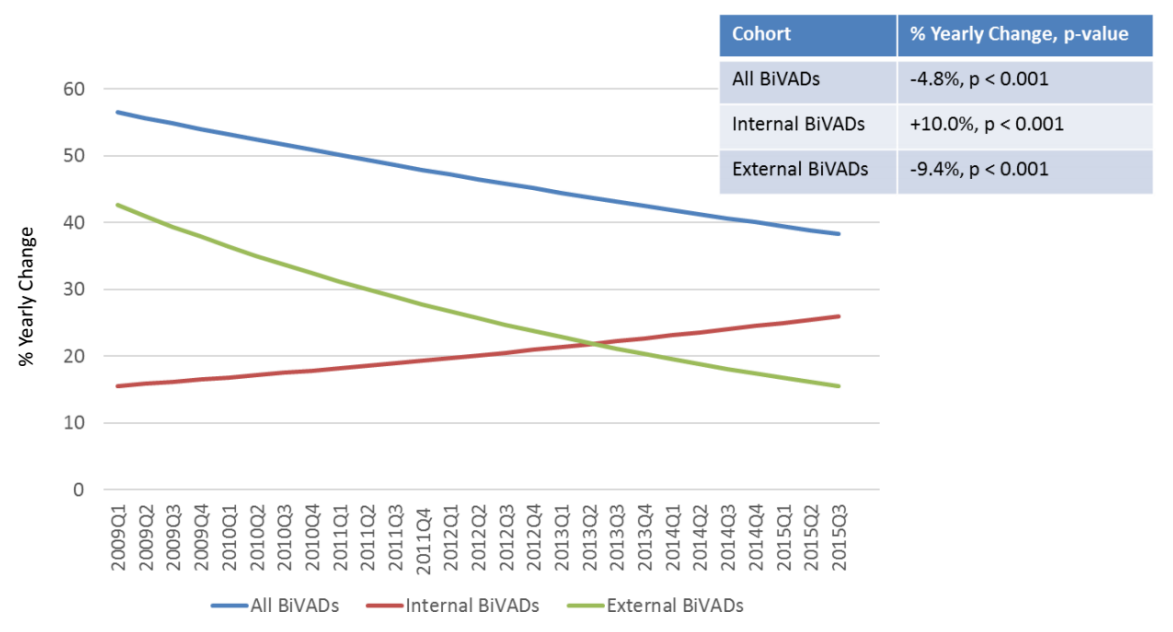




\begin{tabular}{|l|l|}
\hline Total & 0.083 \\
\hline Deceased & $.002(+52 \% / y r)$ \\
\hline Alive & 0.738
\end{tabular}

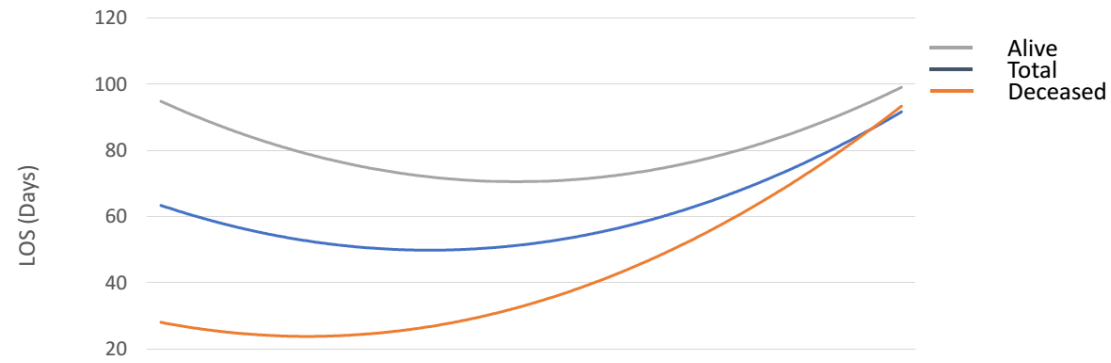

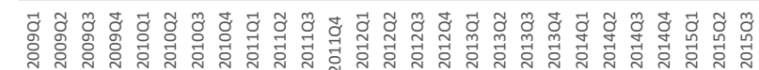

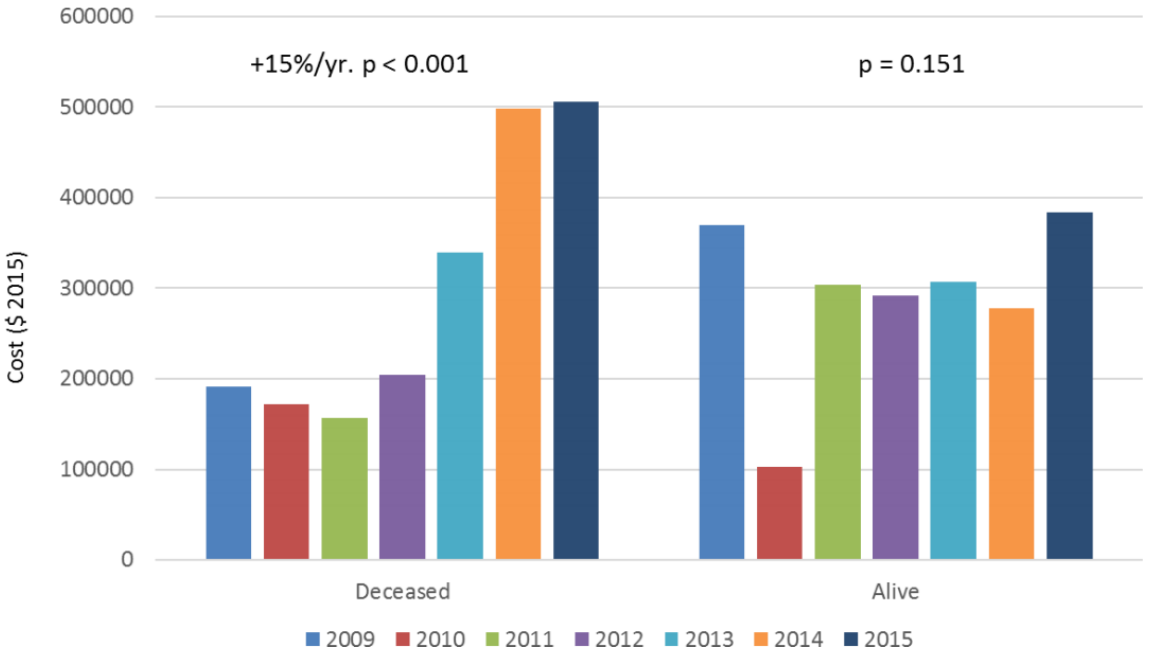




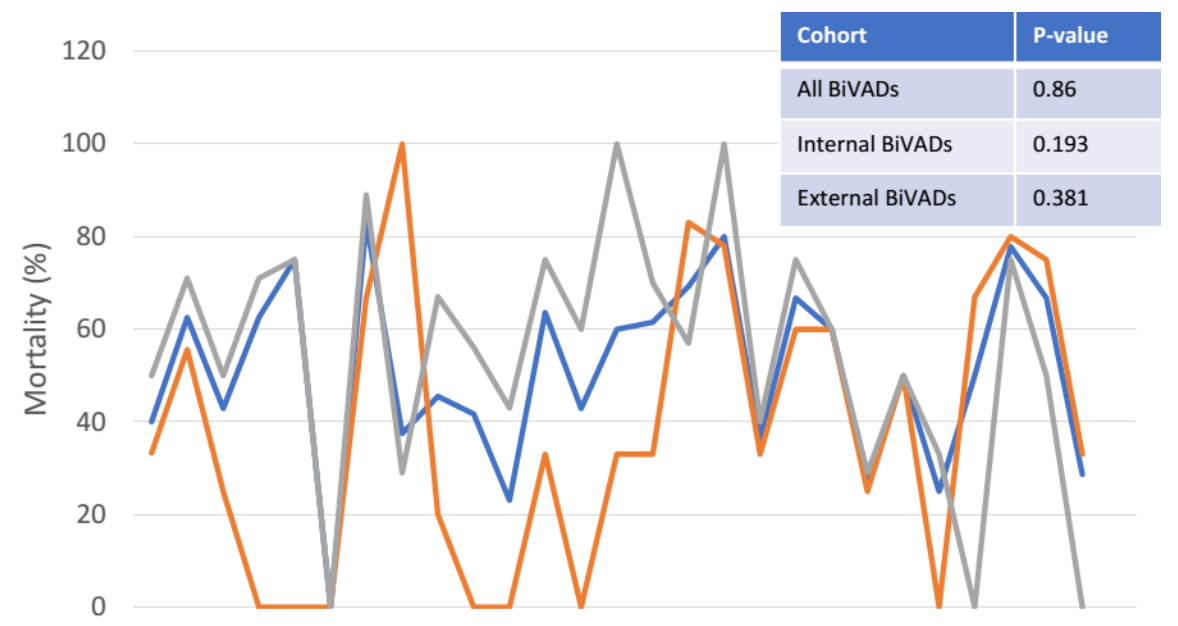

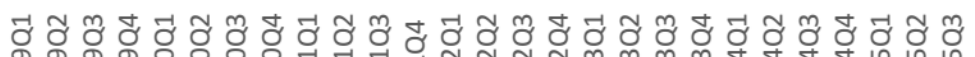

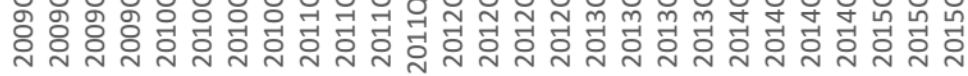

—All BiVADs —Internal BiVADs -External BiVADs 